\title{
Power Bayesian Markov Chain Monte Carlo (MCMC) for Modelling Extreme Temperatures in Sumatra Island Using Generalised Extreme Value (GEV) and Generalised Logistic (GLO) Distributions
}

\author{
Rado Yendra ${ }^{1 *}$, Ibrahim Sulaiman Hanaish ${ }^{2}$, Ahmad Fudholi $^{3,4}$ \\ ${ }^{1}$ Department of Mathematics, Faculty of Science and Technology, Universitas Islam Negeri Sultan Syarif Kasim Riau, \\ Pekanbaru 28293, Indonesia \\ ${ }^{2}$ Department of Statistics, Faculty of Science, Misurata University, Misurata 2478, Libya \\ ${ }^{3}$ Solar Energy Research Institute, Universiti Kebangsaan Malaysia, Bangi 43600, Selangor, Malaysia \\ ${ }^{4}$ Research Centre for Electrical Power and Mechatronics, Indonesian Institute of Sciences (LIPI), Bandung 40135, Indonesia
}

Corresponding Author Email: rado.yendra@uin-suska.ac.id

https://doi.org/10.18280/mmep.080305

Received: 10 March 2021

Accepted: 6 May 2021

\section{Keywords:}

MCMC, extreme value distribution, generalised

logistic distribution, maximum temperature

\begin{abstract}
Climate projections suggest that the frequency and intensity of some environmental extremes will be affected in the future because of climate change. Climate change has brought about new, unprecedented weather patterns, including changes in extreme temperature. Ecosystems and various sectors of human activities are sensitive to high and low temperatures, especially when these occur over extended periods. Sumatra Island is part of the Indonesian state, where most provinces are trough by tropical climates and have annual maximum daily temperatures varying from $72^{\circ} \mathrm{F}-97^{\circ} \mathrm{F}$. This study focuses on the reduction and management of the disaster risk that occurs as a result of extreme high temperatures that lead to global change and heat waves. The main goal of this study is to find the best-fitting distribution to extreme daily temperatures measured over the 12 stations on Sumatra Island in 1999-2019 by using the power of Bayesian Markov Chain Monte Carlo (MCMC) approach. The study also predicts the extreme temperatures for the next 10, 50 and 100 years through return periods. In this study, extreme temperature events are defined by methods based on the annual maximum of daily temperature. Generalised extreme value (GEV) and generalised logistic (GLO) distributions are fitted to data corresponding to the methods to describe the extremes of temperature and predict its future behaviour. Graphical inspection [distribution function (cdf)] and numerical criteria [root mean square error (RMSE)] are used to select the most suitable model. In most cases, graphical inspection gives similar results but the RMSE results differ. Finally, we find evidence that suggests most regions (S1, S3, S5, S6, S7, S8, S9, S10, S11 and S12) have a GEV distribution, which provides the most appropriate model for the annual maximums of daily temperatures, while the GLO distribution gives the reasonable model for the daily temperature data for the S2 and S4 locations. Furthermore, estimates of 10-, 50- and 100-year return levels for extreme temperatures are derived on the basis of the identified model.
\end{abstract}

\section{INTRODUCTION}

Extreme weather events amplified by climate change can lead to major environmental issues affecting human society. Over the past two decades, a major component of climate change which has been analysed extensively is temperature [1, 2]. In high-temperature days, people switch on their cooling systems until the point at which all cooling systems are operating, resulting in an extreme increase in electricity demand [3]. To this effect, modelling the occurrence of extreme high temperatures is vital in the energy sector. Steffen et al. [4] emphasized that heat waves are contributing factors towards the occurrence of several natural disasters, which affect economies and the lives of people worldwide. Heat waves has several disastrous impacts, which are highlighted in this study. The presence of extreme heat leads to drought and health consequences, which account for numerous cases of hospitalisations and deaths [4-7]. When heat waves occur, a high demand for water transpires because water reservoirs dwindle, and water is used to guard against the risk of fire in various situations [4-7]. Civil constructions, such as roads and buildings, also become at risk of collapsing because of extreme heat, thereby endangering people's lives [4-7]. Agricultural sectors face severe challenges in the presence of extreme heat, to the extent that livestock starve and perish because of drought and health challenges arising because of extreme heat [4-7]. Thus, an accurate and precise statistical modelling of the occurrence of extreme high temperatures is necessary for the purpose of guarding against the risk of such disastrous impacts occurring because of hot spells or heat waves.

Most classical statistical techniques frequently used in the energy sector and in meteorological analysis are classified into regression analysis, time series, state space and Kalman filtering [8]. The limitation commonly encountered among such techniques is that they concentrate on the mean instead 
of the tails of the distributions. This feature leads to unreliable estimates because most of the sample values fall outside the tails of the distributions, adding to the difficulty of estimating the model parameters that would lead to a good fit in the tails [9-12]. The problems that arise as consequences of using statistical techniques that do not concentrate on the tails of the distributions are overcome by the use of extreme value theory (EVT), which can model the asymptotic behaviour of thin- or heavy-tailed distributions [13]. According to Hyndman and Fan [13], the frequency of the occurrence of coldest or hottest temperatures is an extreme event and is best modelled with the use of EVT. In the past two decades, EVT has been applied in numerous studies on climate variables, generally temperature and precipitation [14-17]. The studies evaluated generalised extreme value (GEV) distributions in observational data and in output from general circulation models and regional climate models. GEV statistical distribution has been used to examine the time-series of climate extremes. Jarušková and Rencová [17] studied the extreme changes in annual maxima and minima temperature series using five meteorological sites, implementing EVT and hypothesis testing within the framework of the GEV-based method. Most often, the modelling of extreme temperature events was performed using sophisticated distribution modelling methods, such as GEV distribution, which is also known as extreme Frechet and generalised logistic (GLO) distribution.

This study focuses on extreme temperatures in Sumatra, an island of Indonesia. Indonesia has an equatorial climate, which signifies abundant sunshine, generally high heat, high humidity and high rainfall year-round. However, blanket of clouds obstructs a substantial amount of sunshine and, on the average, Indonesia receives about six hours of sunshine per day. Nonetheless, seasonal and spatial variations are present in the amount of sunshine received. Sumatra Island is highly influenced by the surrounding sea and wind system. An increasing trend in the average surface temperature has been observed for Indonesia over the years. The western part of Indonesia where Sumatra Island is located reported a greater rise in temperature compared with other regions in Indonesia, and the months September to November recorded the highest temperature increase. Studies on extreme temperatures are beneficial to our understanding of extreme events. Decision makers, risk management professionals and researchers in climatology will benefit from acquired knowledge on the behaviours of extreme temperatures, as appropriate policies and plans can be drawn to prepare the general public for changes due to extreme temperatures. The objective of this study is to quantify and describe the behaviours of extreme temperatures in Sumatra Island. Particularly, we aim to model extreme temperatures using GEV and GLO distributions. The estimation parameters of these distributions are determined using Bayesian non-informative prior use of Bayesian Markov Chain Monte Carlo (MCMC) techniques. The basic theory of Bayesian analysis of extreme values is well established and is presented in a number of excellent articles and texts, such as those by Coles, S. et al. [18, 19]. Bayesian MCMC techniques can avoid highly complex mathematical calculations in estimating parameters. The power of these techniques lies in their ability to produce parameters that converge in iterations performed many times; they can also easily use certain initial values in the iteration process carried out. Bayesian MCMC techniques have a fundamental impact on virtually every statistical methodology. Bayesian analysis has enormous potential for various research fields. The theoretical background to apply non-informative prior distribution is plentiful [20-22], and extensive literature is available on the theory behind MCMC techniques and their applications. Introductions to the area are provided by the authors [23-25]. From the best-fitted distribution, the return period can be generated for 10-, 50- and 100-year return levels, and the results based on the two distributions can be compared.

\section{DATA AND AREA STUDY}

The data consisting of daily temperatures (in Fahrenheit) from 12 stations in Sumatra Island from 1999 to 2019 are obtained from meteorological, climatological and geophysical agencies. The descriptive statistics for daily temperatures from these stations are presented in Table 1, and annual maximum daily temperatures are presented in Figure 1. The 12 stations with codes shown in Table 1 represent 10 provinces located on Sumatra Island.

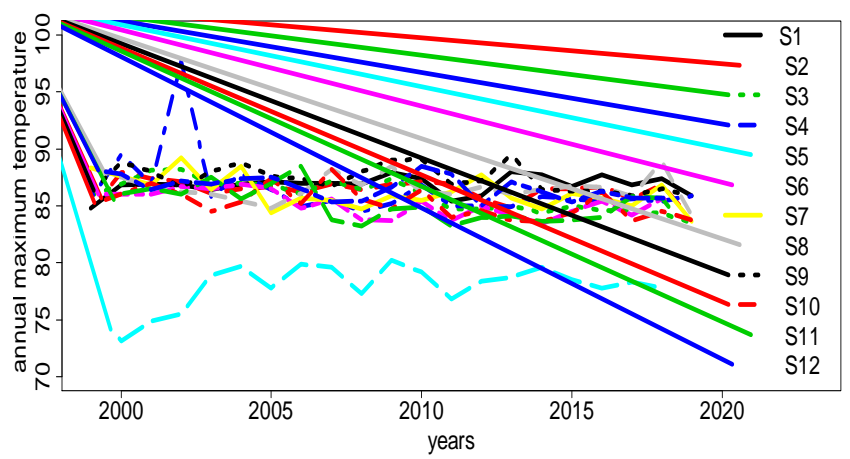

Figure 1. Annual maximum daily temperature data

Table 1. Main characteristics and codes of the 12 stations

\begin{tabular}{ccccccc}
\hline Station & Code & Minimum & Mean & Median & Variation & Maximum \\
\hline Belawan & S1 & 73.90 & 82.14 & 82.10 & 3.41 & 88.00 \\
Cut bau maimun & S2 & 73.00 & 81.37 & 81.50 & 4.71 & 87.90 \\
Cut nyak dien & S3 & 73.50 & 81.23 & 81.40 & 4.45 & 88.20 \\
Dabo singkep & S4 & 72.90 & 81.16 & 81.40 & 4.57 & 97.70 \\
Depati parbo & S5 & 62.80 & 72.96 & 73.00 & 3.09 & 80.20 \\
Fatmawati & S6 & 72.30 & 80.45 & 80.60 & 3.69 & 86.90 \\
Raden intan & S7 & 73.30 & 80.79 & 80.80 & 4.01 & 89.20 \\
Sultan Mahmud badarudin & S8 & 71.50 & 81.34 & 81.40 & 4.32 & 88.90 \\
Sultan syarif kasim & S9 & 73.80 & 81.90 & 81.90 & 5.06 & 89.50 \\
Tabing padang & S10 & 69.90 & 80.31 & 80.50 & 3.41 & 88.20 \\
Pangkal pinang & S11 & 73.00 & 80.77 & 80.80 & 4.59 & 90.10 \\
Hang nadim & S12 & 73.20 & 81.72 & 81.90 & 4.46 & 88.50 \\
\hline
\end{tabular}


On the basis of data in Table 1, temperatures in Sumatra Island can be classified into hot temperatures. The data likewise reveal no significant difference between the stations used in this study. The homogeneity of variation values can also be interpreted that Sumatra Island has a temperature characteristic of almost the same temperature. The maximum annual temperature data, as shown in Figure 1, also indicate that Sumatra Island experiences rather high temperatures, ranging from $70^{\circ} \mathrm{F}-100^{\circ} \mathrm{F}$. These results show that many natural phenomena can occur in the extreme temperature range, including drought springs and decreased yields due to drought. For this reason, determining the best model to describe the characteristics of extreme temperatures for the next 10, 50 and 100 years is imperative.

\section{METHODOLOGY}

\subsection{Probability distribution}

The most common analysis of extreme hydrological events involves the use of annual maximum or annual extreme. Hence, the series obtained will have a length equal to the number of years. Two probability distributions associated with modelling extreme events are considered in this study, namely GEV and GLO. The probability density function, probability function and quantile function for each distribution considered are shown in Table 2, where $\mathrm{x}$ denotes the observed values of the random variable representing the event of interest, $\alpha$ is the scale parameter, $\varepsilon$ is the location parameter and $\kappa$ is the shape parameter. To fit a particular theoretical distribution to the observed distribution of annual maximum temperatures, we estimate the parameters using Bayesian MCMC techniques.

\subsection{Bayesian MCMC and goodness-of-fit tests}

This section introduces the idea of Bayesian MCMC using non-informative priors. Suppose that prior beliefs about $\theta$ can be formulated and expressed by a probability density function $\pi(\theta)$ with no reference to the data. The likelihood for $\theta$ is $L(\theta \mid x)$. The prior information and the likelihood can be combined using Bayes theory to produce a posterior distribution for $\theta$ as follows:

$$
\pi(\theta \mid x)=\pi(\theta) L(\theta \mid x) / \int \pi(\theta) L(\theta \mid x) d \theta
$$

The usual function of an extreme value analysis is to describe the extreme behaviours of an observed process to find the probability of extreme events occurring in the future. Within the Bayesian framework, prediction is possible through the predictive distribution. Let $y$ denote a future observation with probability density function $f(y \mid \theta)$. Then,

$$
f(y \mid x)=\int f(y \mid \theta) \pi(\theta \mid x) d \theta
$$

$f(y \mid x)$ is the predictive distribution of $y$ given $\mathrm{x}$. Thus, if a suitable prior distribution can be specified, there are good reasons to choose Bayesian procedures. The difficulty in computing the integral in predictive distribution means the simulation techniques, such as Bayesian MCMC, can be overcome to simulate realisations of the posterior distribution. The main problem in Bayesian MCMC with non-informative prior distribution is that the priors are constructed by assuming no information is available about the process apart from the data. In this study, prior density is chosen to be:

$$
\begin{aligned}
& \text { with } \varphi \\
& =\log \alpha, \varphi \sim N(0,100), \xi \sim N(0,1000), \text { and } \kappa \sim N(0,10)
\end{aligned}
$$

The variances chosen are large enough to make the distribution almost flat, corresponding to prior ignorance. Thus, the posterior density is:

$$
\pi(\varphi, \xi, \kappa \mid y) \infty \pi(\varphi, \xi, \kappa) L(\varphi, \xi, \kappa \mid y)
$$

The full details of the algorithm are as follows:

1. Initialise the chain $\theta^{0}=\left(\varphi^{0}, \xi^{0}, \kappa^{0}\right)$ and the counter at $j=1$.

2. Put $\varphi^{*}=\varphi^{(j-1)}+\omega_{\varphi}, \omega_{\varphi} \sim N(0,4)$.

3. Accept $\varphi^{(j)}=\varphi^{*}$ with probability $a\left(\varphi^{(j-1)}, \varphi^{*}\right)=$ $\min \{1, A\}$, where,

$A=\frac{\pi\left(\varphi^{*} \mid \xi^{(j-1)}, \kappa^{(j-1)}\right)}{\pi\left(\varphi^{(j-1)} \mid \xi^{(j-1)}, \kappa^{(j-1)}\right)}$ and $\varphi^{(j)}=\varphi^{(j-1)}$ otherwise.

4. Put $\xi^{*}=\xi^{(j-1)}+\omega_{\xi}, \omega_{\xi} \sim N(0,0.3)$.

Table 2. List of distributions used in this study

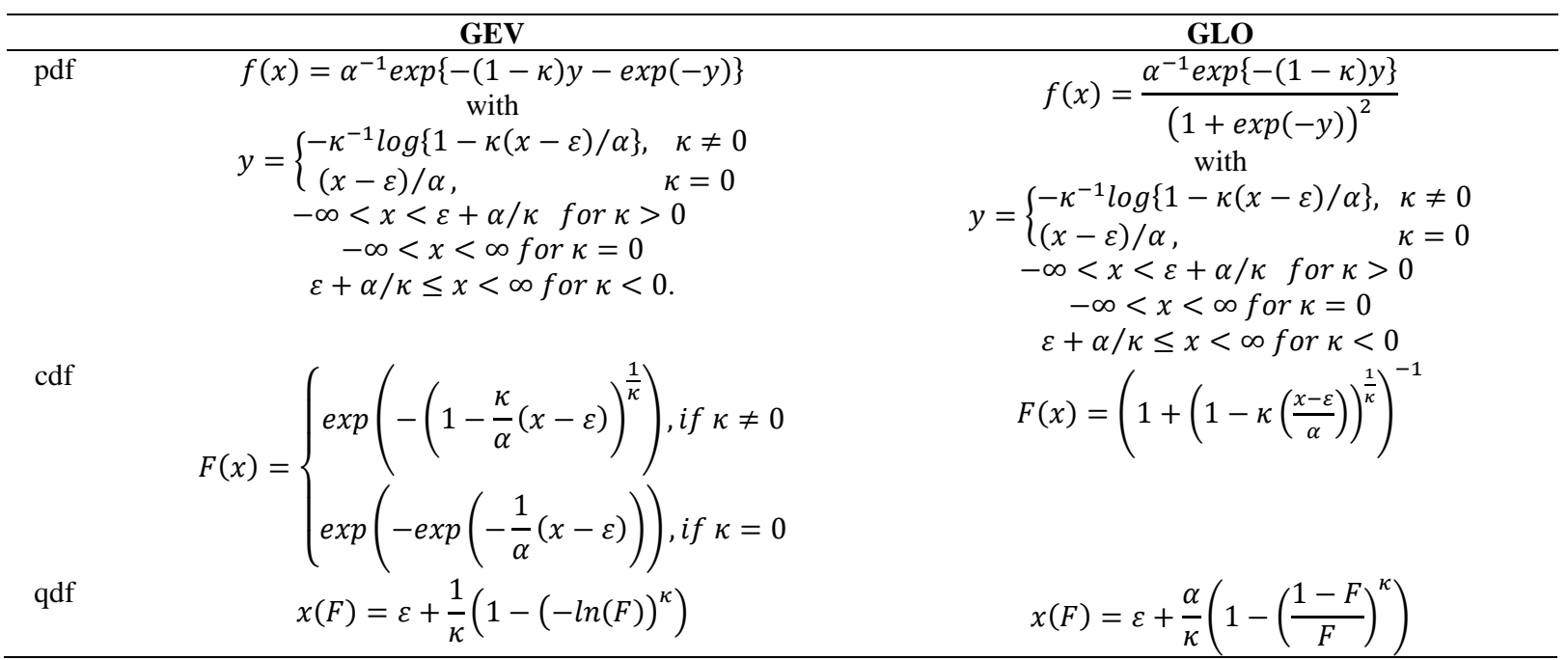


5. Accept $\xi^{(j)}=\xi^{*}$ with probability $a\left(\xi^{(j-1)}, \xi^{*}\right)=$ $\min \{1, A\}$, where,

$A=\frac{\pi\left(\xi^{*} \mid \phi^{(j)}, \kappa^{(j-1)}\right)}{\pi\left(\xi^{(j-1)} \mid \phi^{(j)}, \kappa^{(j-1)}\right)}$ and $\xi^{(j)}=\xi^{(j-1)}$ otherwise

6. Put $\kappa^{*}=\kappa^{(j-1)}+\omega_{\kappa}, \omega_{\kappa} \sim N(0,0.1)$.

7. Accept $\kappa^{(j)}=\kappa^{*}$ with probability $a\left(\kappa^{(j-1)}, \kappa^{*}\right)=$ $\min \{1, A\}$, where,

$$
A=\frac{\pi\left(\kappa^{*} \mid \phi^{(j)}, \xi^{(j)}\right)}{\pi\left(\kappa^{(j-1)} \mid \phi^{(j)}, \xi^{(j)}\right)} \text { and } \kappa^{(j)}=\kappa^{(j-1)} \text { otherwise }
$$

8. Increase counter from $j$ to $j+1$ and return to Step 2 .

Once the distribution of the observed values is determined for annual maximum temperature series, expected frequencies under the assumed distribution are computed for each station. The most appropriate distribution for each station is identified using the results found according to several goodness-of-fit (GOF) tests.

The GOF tests considered are root mean square error (RMSE). The methods involve assessing the difference between observed and expected values under the assumed distribution. The formulas for the tests are:

$$
R M S E=\sqrt{\frac{1}{n} \sum_{i=1}^{n}\left(\frac{x_{i: n}-\hat{Q}\left(F_{i}\right)}{x_{i: n}}\right)^{2}}
$$

where, $x_{i: n}$ is the observed values for $i$ th order statistics of random sample of size $n$, and $\bar{Q}\left(F_{i}\right)=\frac{1}{n} \sum_{i=1}^{n} \hat{Q}\left(F_{i}\right)$ is the estimated quantile values associated with the Gringorton plotting position $F_{i}$. The smallest value of RMSE will indicate the best-fitting distribution.

\section{RESULTS}

The parameters in the two probability distributions, namely GEV and GLO distributions, are estimated using Bayesian MCMC techniques with the random walk chain algorithm. In the case of Bayesian framework, the algorithm in 3.2 can be used to obtain parameters. Daily temperature data for 12 stations will be analysed using the algorithm in 3.2. In each case, 30,000 iterations of the algorithm are carried out. The Bayesian MCMC trace plots and estimated posterior densities for GEV and GLO parameters for 12 stations are given in Tables 3 to 10 accordingly. To validate that the chains converge to the correct place, the same algorithm is carried out using the starting points. The chains for the 12 stations all converge suitably within the first 10,000 iterations. Therefore, the developed proposal distribution works well. The MCMC method used in Bayesian with non-informative prior distribution guarantees that all initial values used can produce an accurate estimate. In Tables 3 to 6 , the initial values used are the same for the GEV and GLO distributions. The same initial value is also used for all stations used in this study.

Table 3. Trace plots of the GEV parameters using Bayesian MCMC techniques for S1-S6

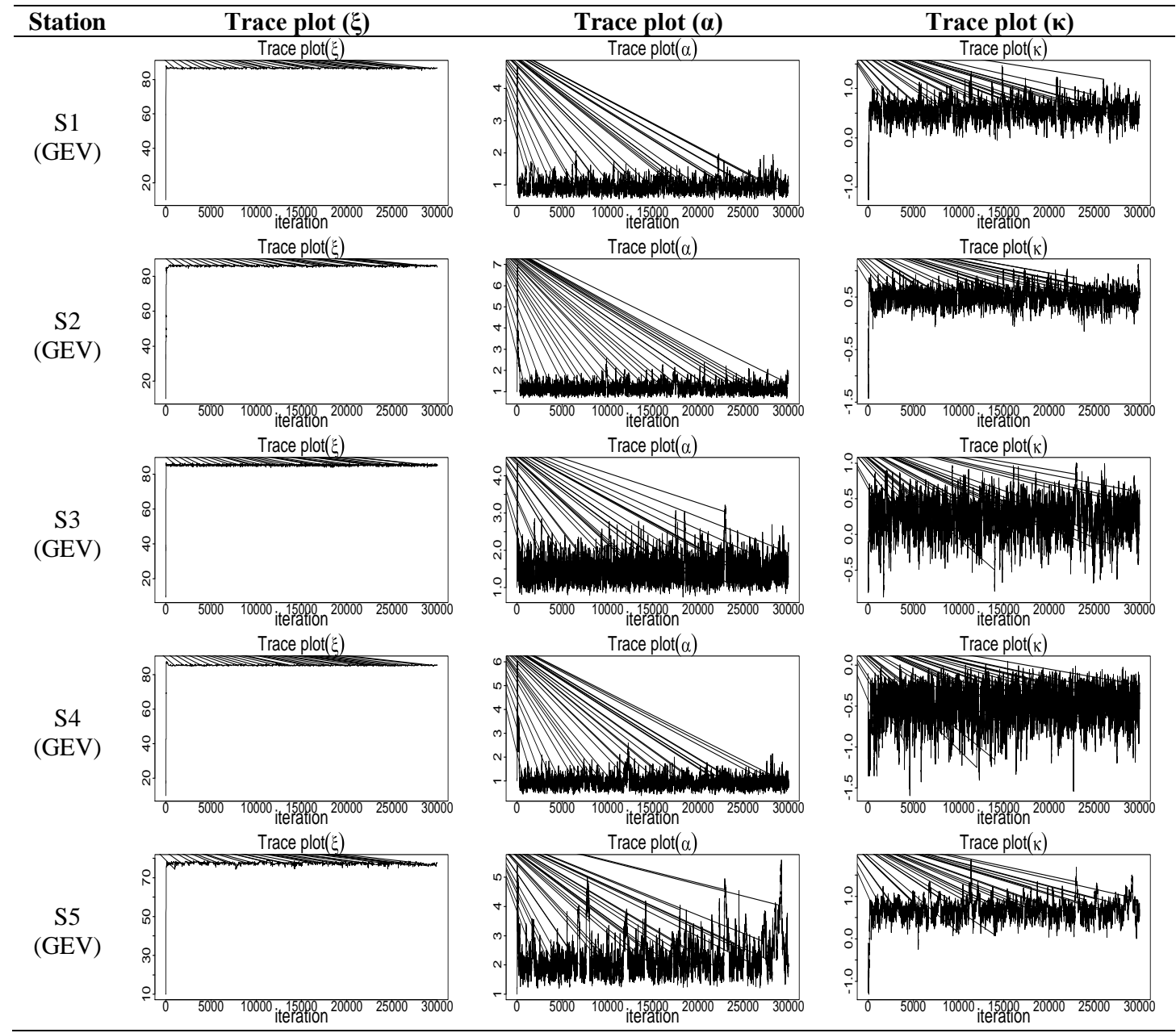



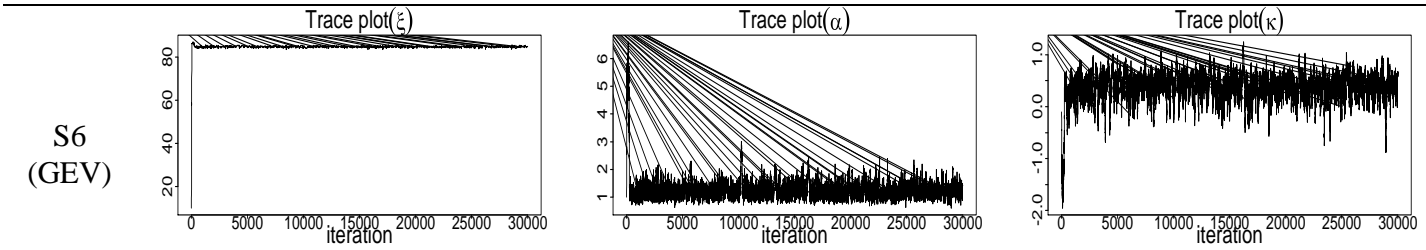

Table 4. Trace plots of the GEV parameters using Bayesian MCMC techniques for S7-S12

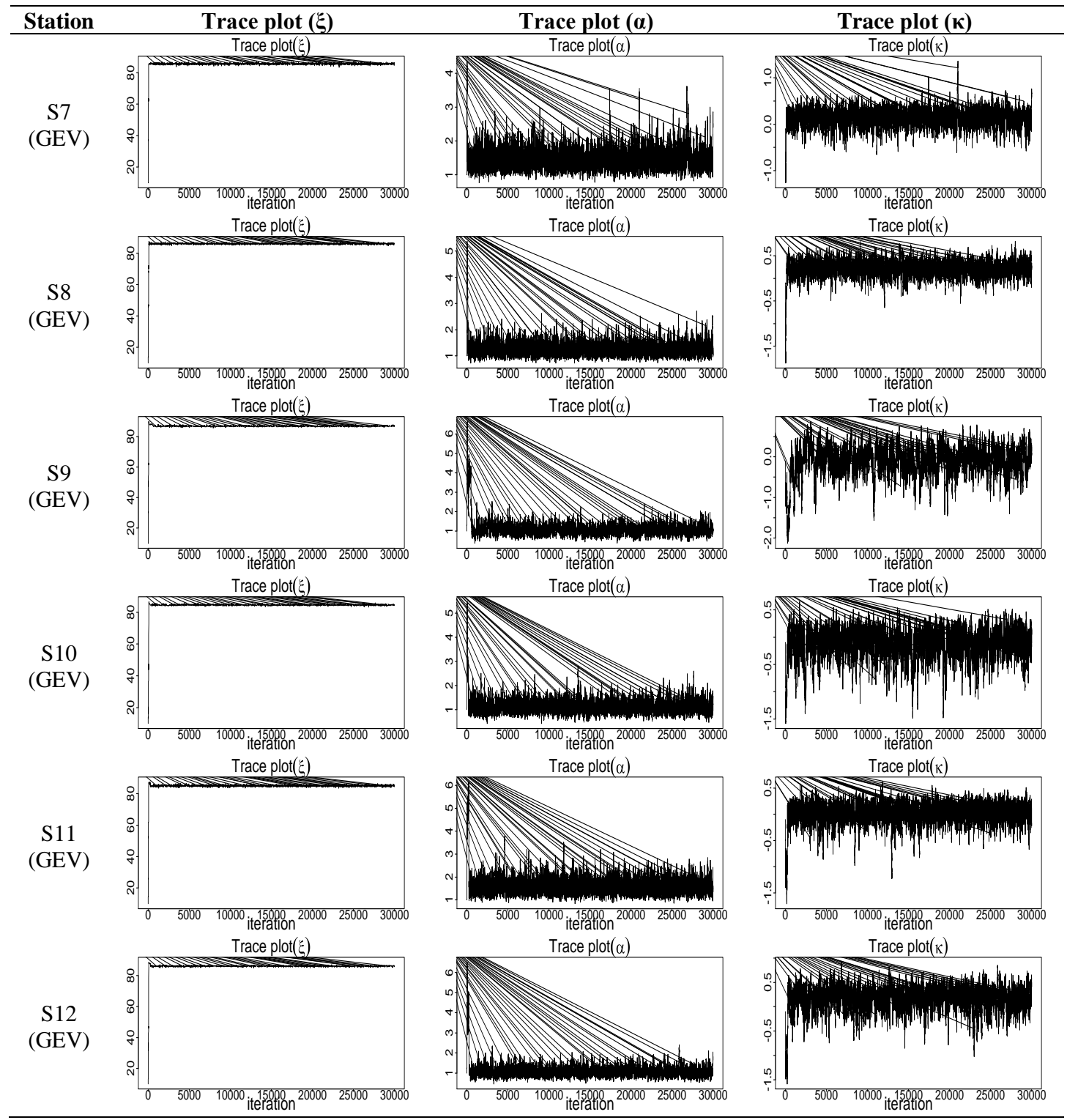

Table 5. Trace plots of the GLO parameters using Bayesian MCMC techniques for S1-S6

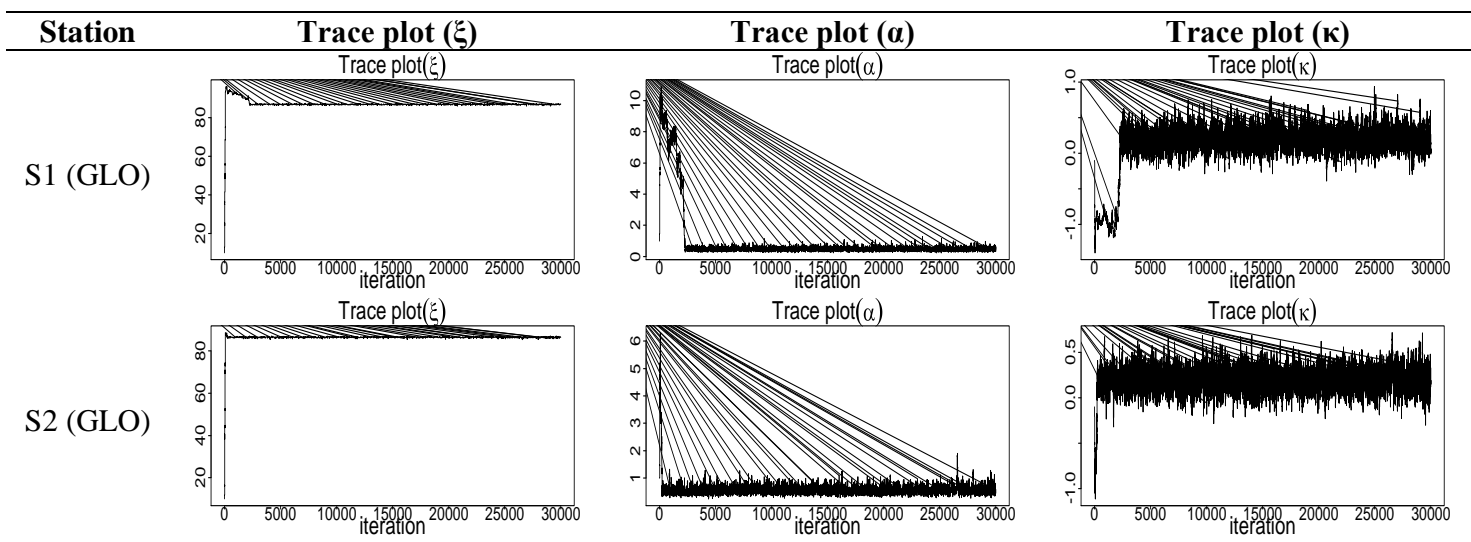




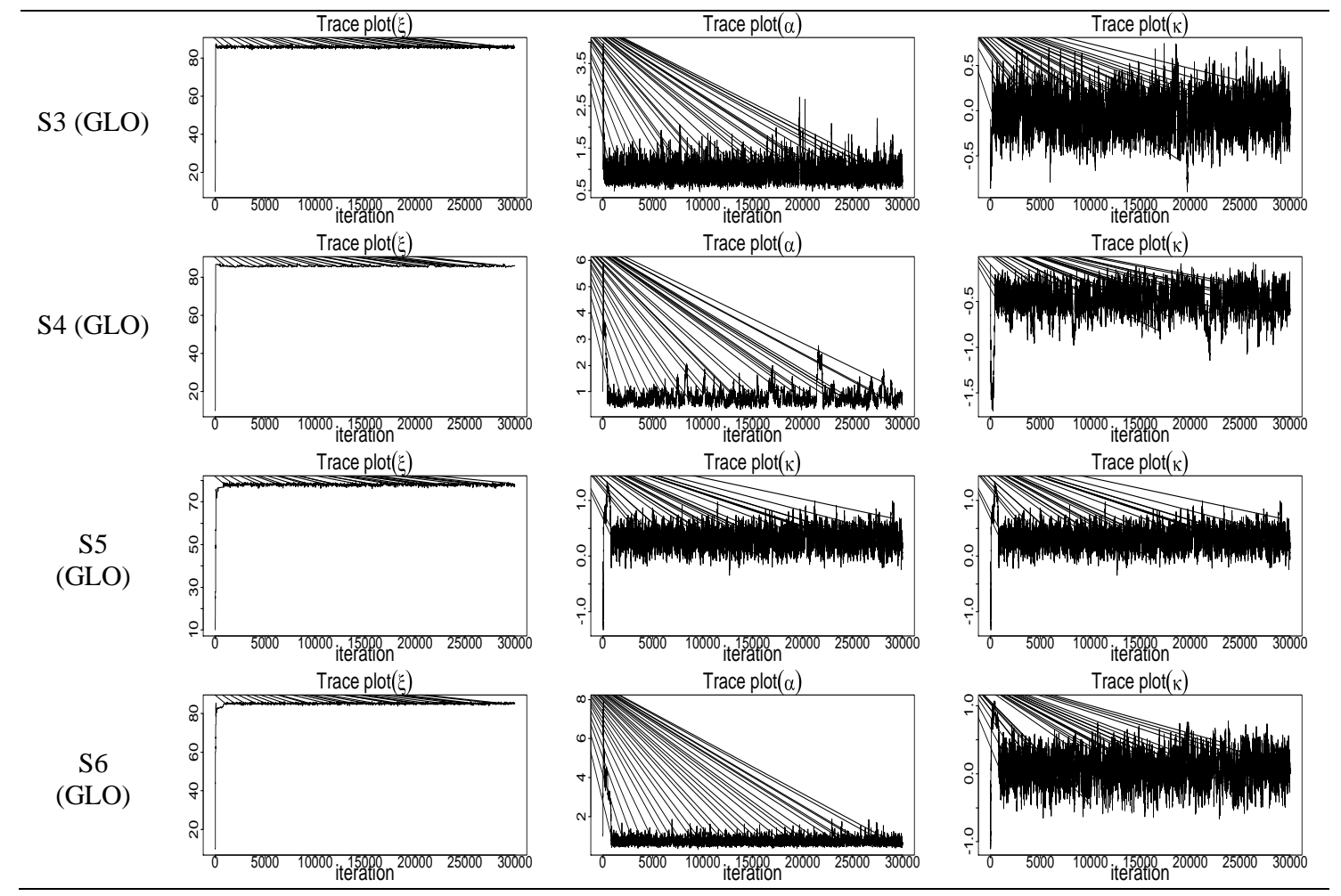

Table 6. Trace plots of the GLO parameters using Bayesian MCMC techniques for S7-S12

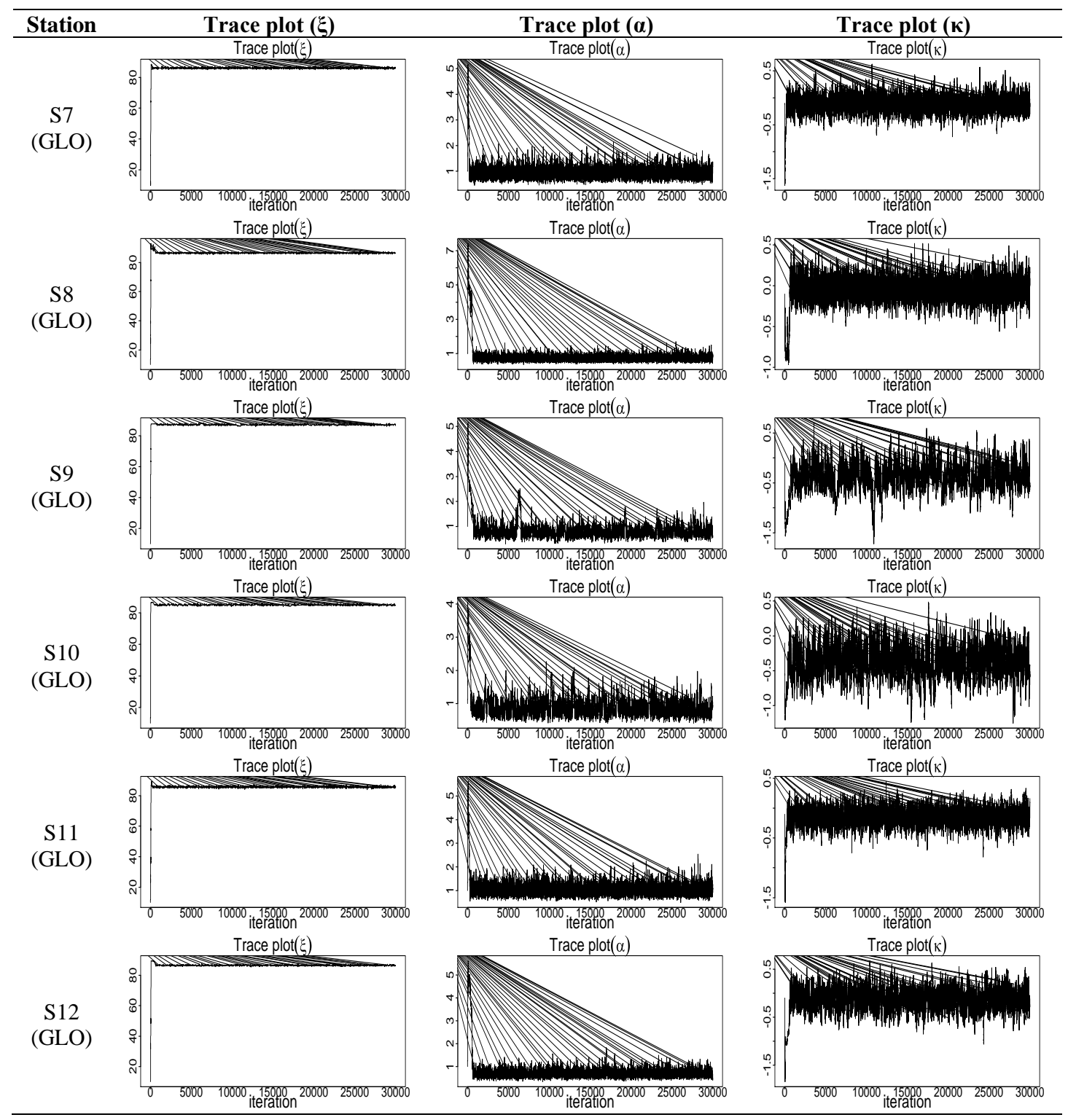


Table 7. Posterior densities of the GEV parameters using Bayesian MCMC techniques for S1-S6

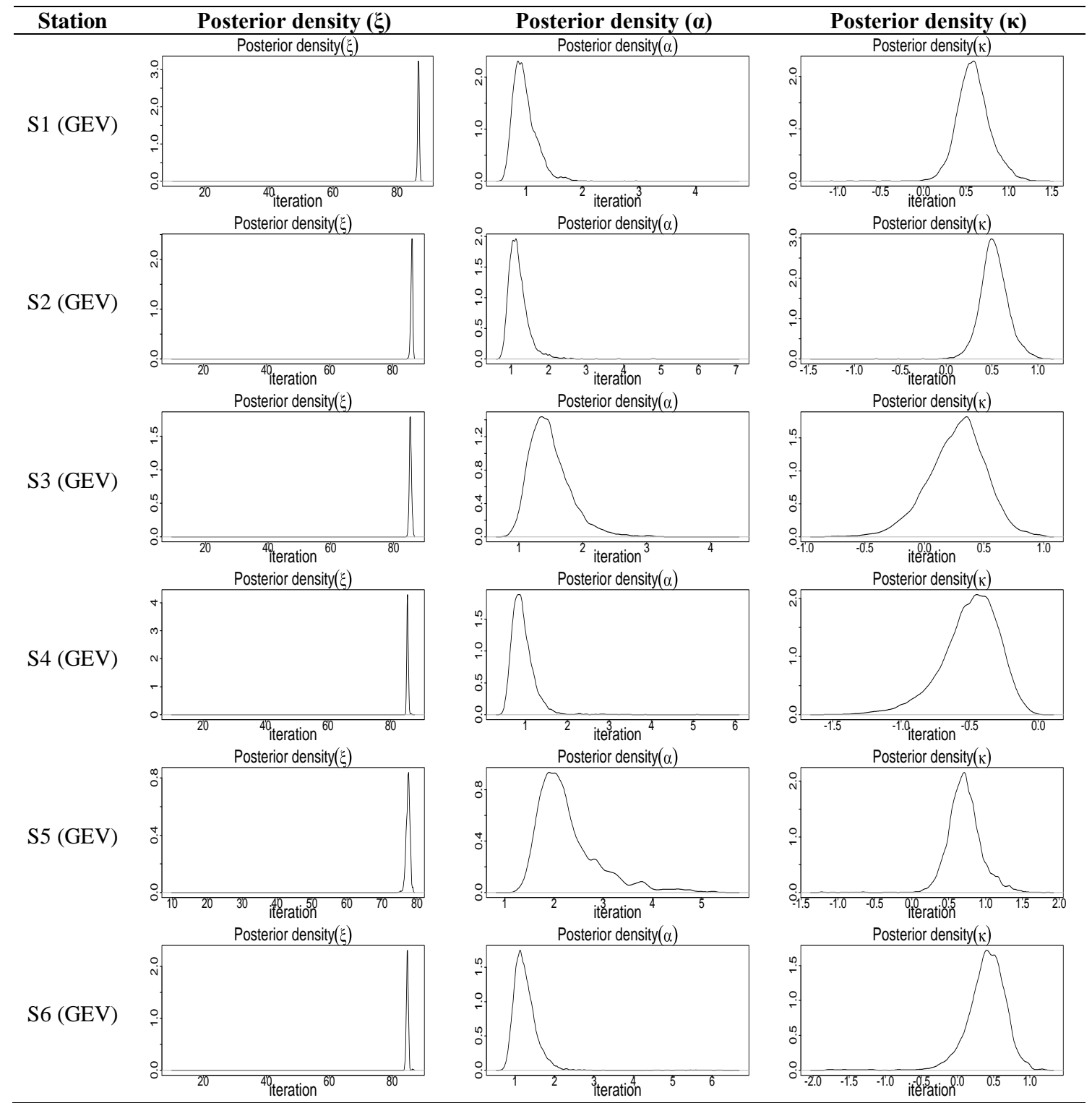

Table 8. Posterior densities of the GEV parameters using Bayesian MCMC techniques for S7-S12

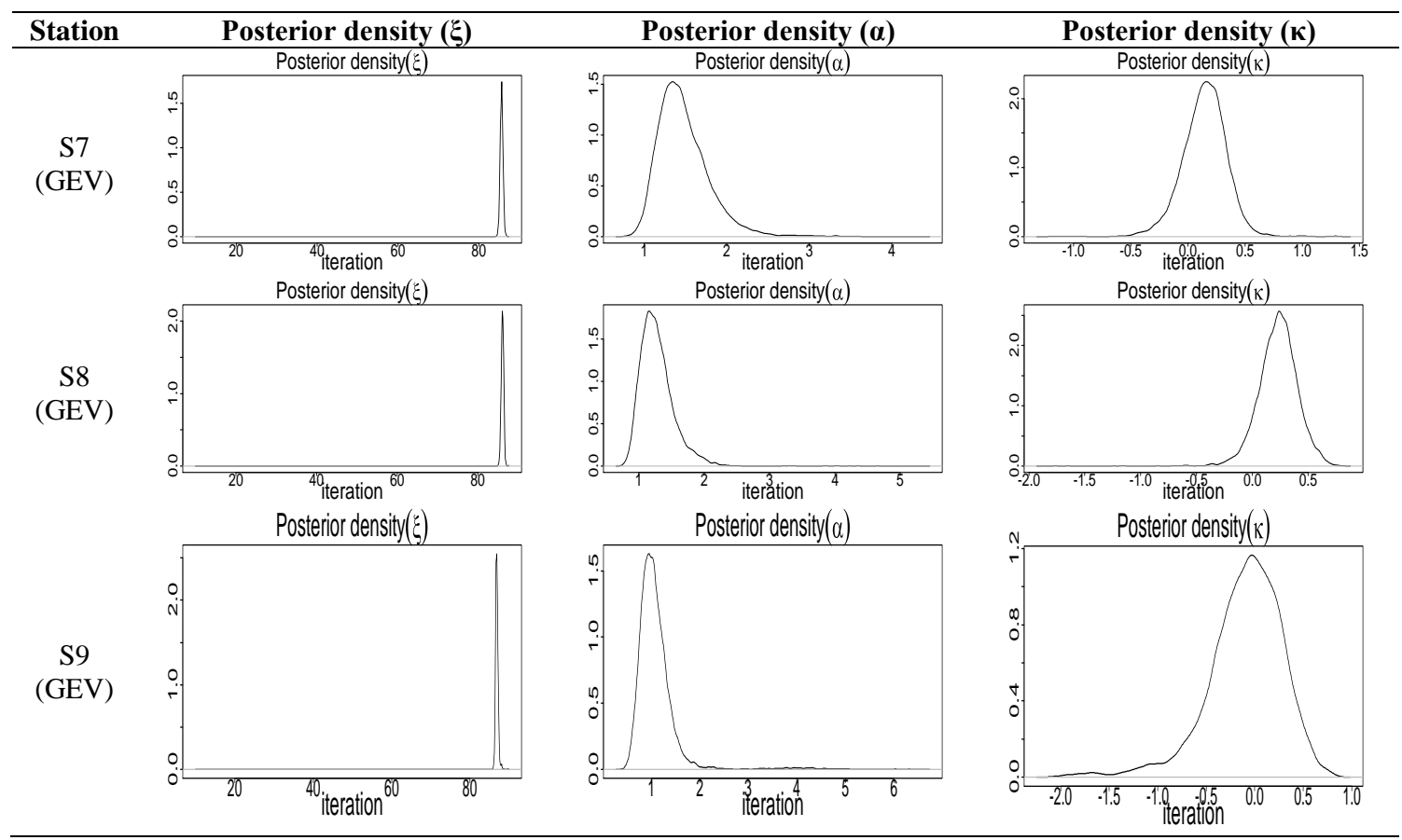




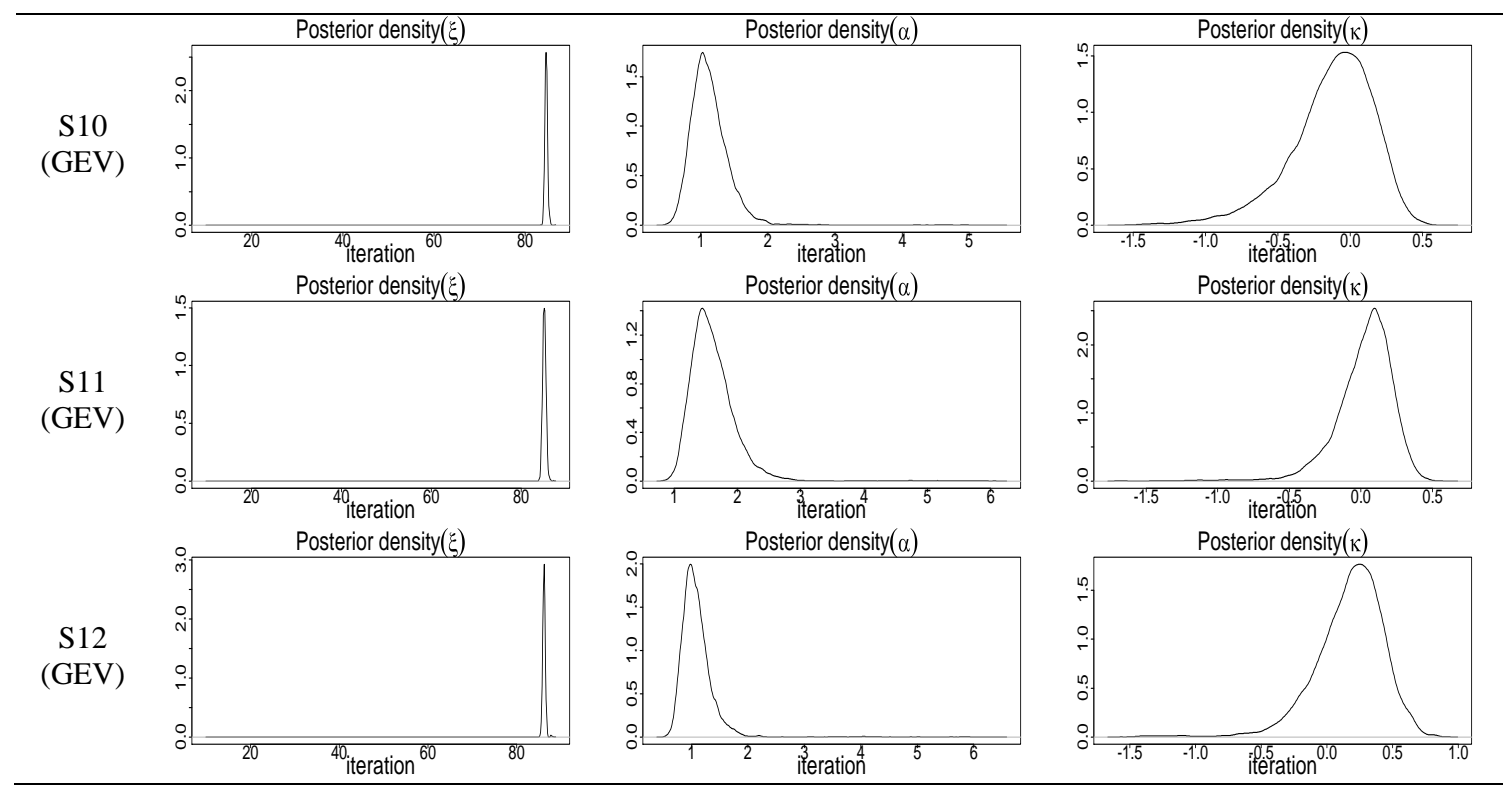

Table 9. Posterior densities of the GLO parameters using Bayesian MCMC techniques for S1-S6

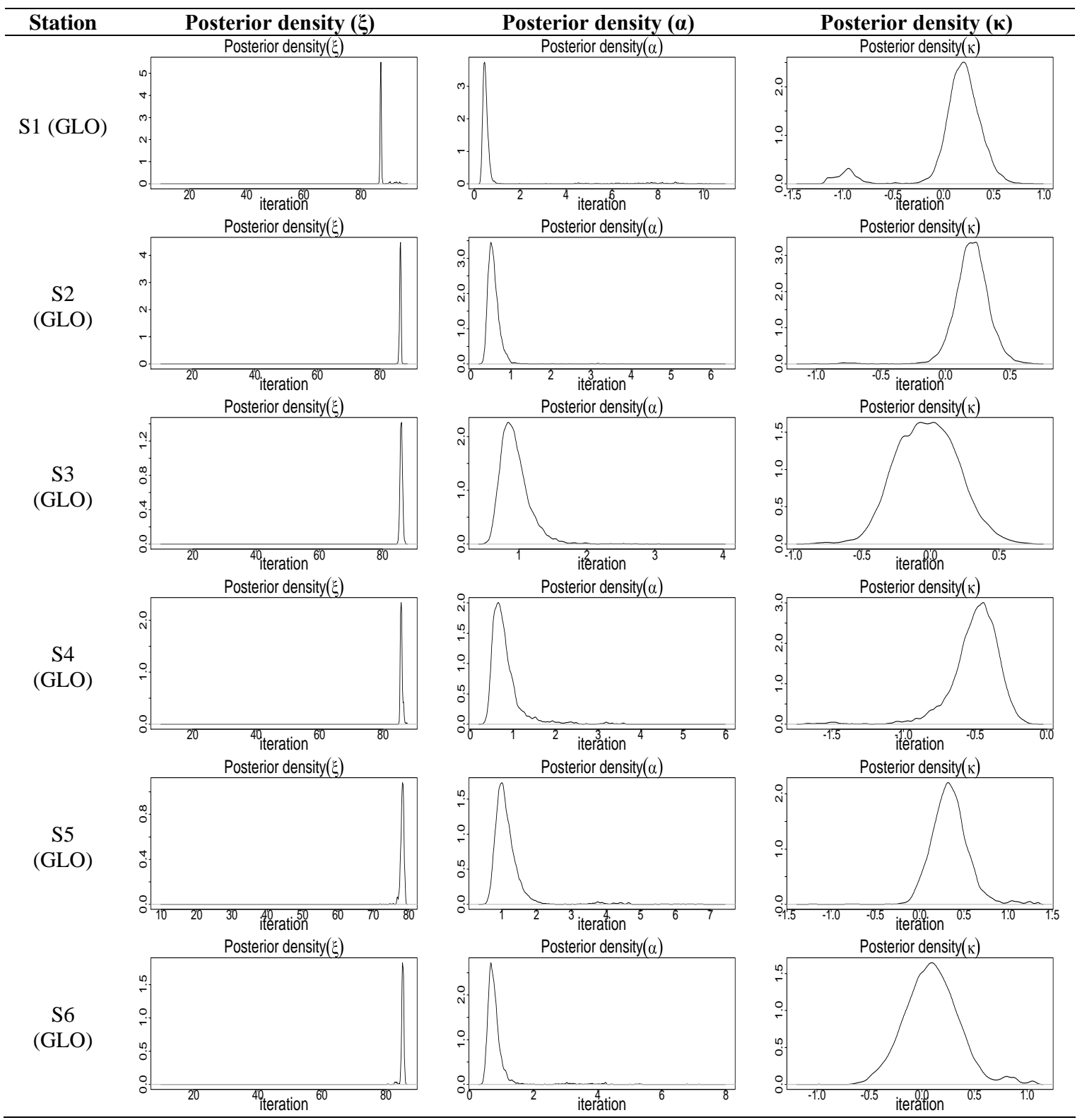


Table 10. Posterior densities of the GLO parameters using Bayesian MCMC techniques for S7-S12
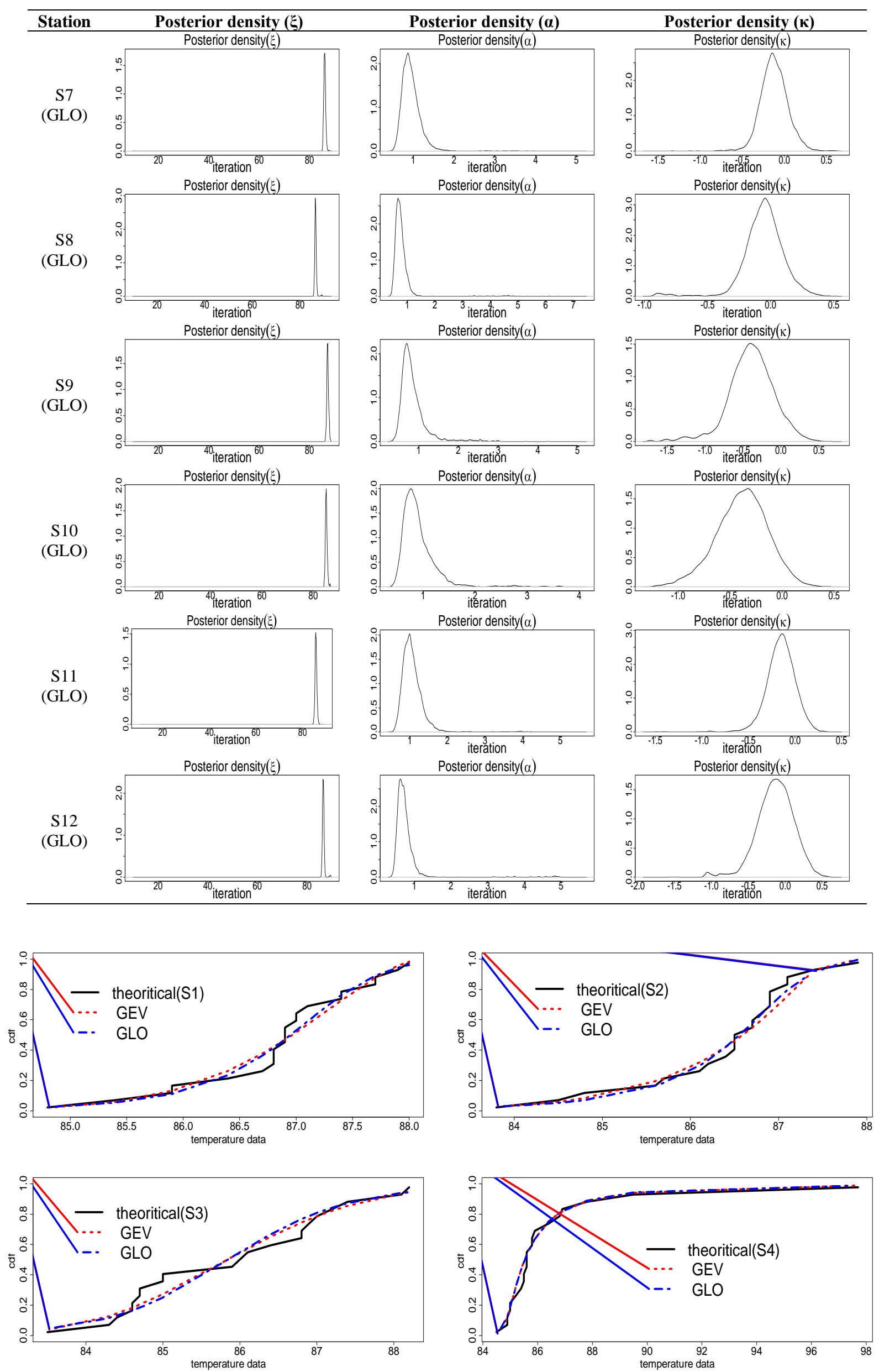

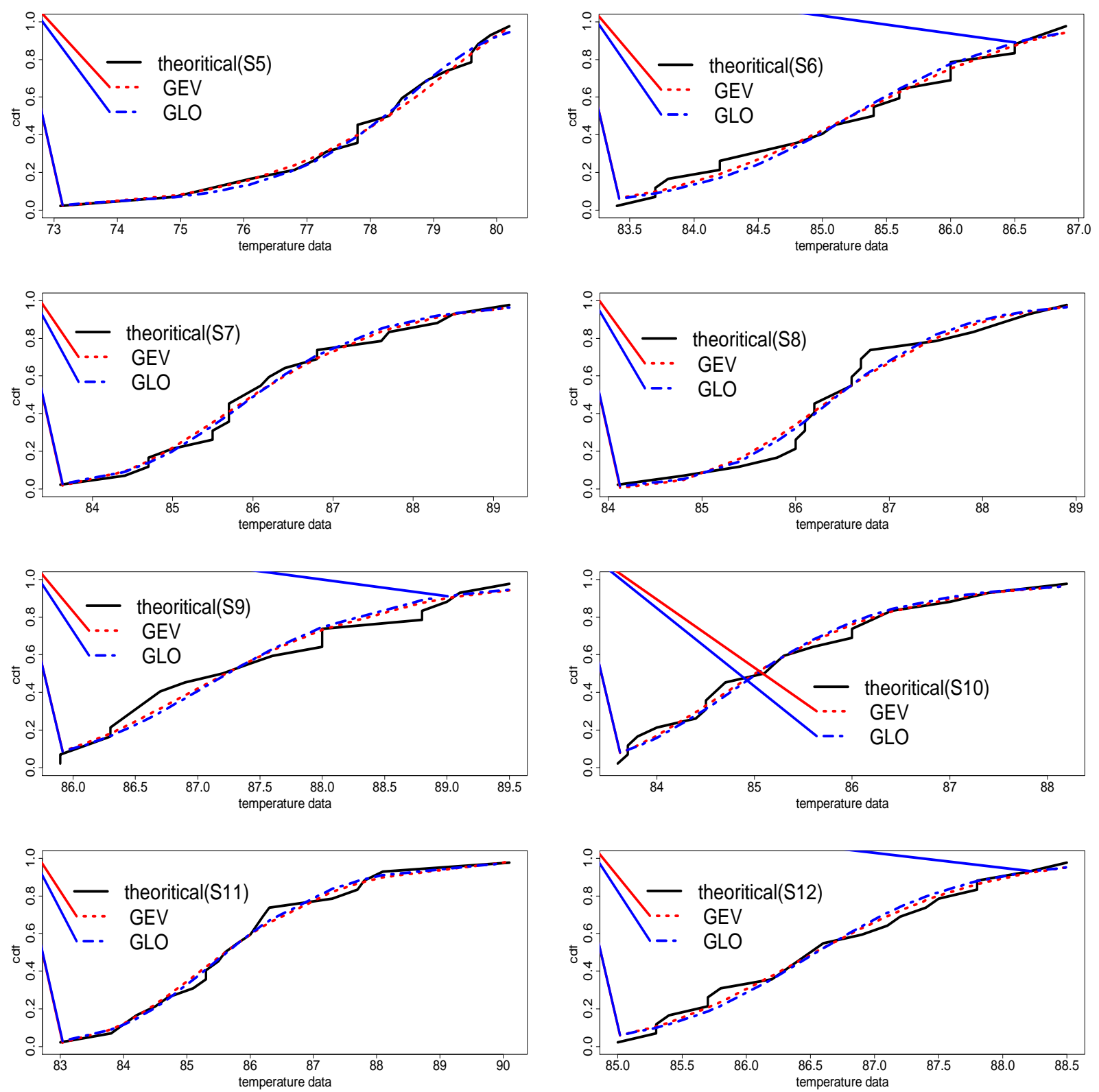

Figure 2. Comparison modelling of GEV, GLO and theoretical distribution function (cdf)

Table 11. Posterior means by Bayesian MCMC for the GEV and GLO parameters and RMSE

\begin{tabular}{ccccccccc}
\hline Station & \multicolumn{4}{c}{ Parameters GEV } & \multicolumn{3}{c}{ Parameters GLO } & \multicolumn{2}{c}{ RMSE } \\
\hline & $(\xi)$ & $(\alpha)$ & $(\kappa)$ & $(\xi)$ & $(\alpha)$ & $(\kappa)$ & GEV & GLO \\
S1 & 86.584 & 0.980 & 0.585 & 86.928 & 0.495 & 0.211 & $\mathbf{0 . 0 0 2}$ & 0.011 \\
S2 & 85.944 & 1.203 & 0.521 & 86.397 & 0.587 & 0.201 & 0.003 & $\mathbf{0 . 0 0 2}$ \\
S3 & 85.319 & 1.489 & 0.269 & 85.816 & 0.950 & -0.025 & $\mathbf{0 . 0 0 4}$ & 0.007 \\
S4 & 85.346 & 0.946 & -0.498 & 85.763 & 0.827 & -0.502 & 0.018 & $\mathbf{0 . 0 1 4}$ \\
S5 & 77.380 & 2.276 & 0.713 & 78.034 & 1.187 & 0.342 & $\mathbf{0 . 0 0 3}$ & 0.006 \\
S6 & 84.806 & 1.281 & 0.392 & 85.093 & 0.842 & 0.096 & $\mathbf{0 . 0 0 3}$ & 0.006 \\
S7 & 85.559 & 1.481 & 0.144 & 86.005 & 0.952 & -0.121 & $\mathbf{0 . 0 0 5}$ & 0.008 \\
S8 & 86.025 & 1.285 & 0.227 & 86.481 & 0.815 & -0.055 & $\mathbf{0 . 0 0 4}$ & 0.006 \\
S9 & 86.795 & 1.104 & -0.096 & 87.159 & 0.854 & -0.389 & $\mathbf{0 . 0 1 1}$ & 0.026 \\
S10 & 84.529 & 1.139 & -0.126 & 85.006 & 0.903 & -0.385 & $\mathbf{0 . 0 1 1}$ & 0.025 \\
S11 & 85.054 & 1.627 & 0.021 & 85.586 & 1.066 & -0.150 & $\mathbf{0 . 0 0 8}$ & 0.009 \\
S12 & 86.103 & 1.104 & 0.169 & 86.503 & 0.777 & -0.133 & $\mathbf{0 . 0 0 4}$ & 0.009 \\
\hline
\end{tabular}

The results of the parameter estimation in the 12 stations using GEV and GLO distributions are summarised in Table 11. In the table, the posterior means of Bayesian MCMC are highly similar to one another regardless of the station and probability distribution. This result proves the power of
Bayesian MCMC to estimate the parameters of GEV and GLO distributions and avoid difficult calculations with the same purpose in estimated parameters. Therefore, Bayesian MCMC techniques have an advantage in estimated parameters. The comparison of the results of the GEV and GLO distributions 
using graphical inspection [distribution function (cdf)] are presented in Figure 2. In most cases, graphical inspection provides the same result, but using numerical criteria, such as RMSE in Table 11, will give the different numerical GOF. Hence, we find the evidence suggesting that, for all GOF tests, most regions (S1, S3, S5, S6, S7, S8, S9, S10, S11 and S12) have a GEV distribution, which provides the most appropriate model for the annual maximums of daily temperatures. By contrast, GLO distribution gives a reasonable model for the daily temperature data for the S2 and S4 locations.

On the basis of the best-fitted models, we can calculate the return values of 10-, 50- and 100-year periods for the 12 stations, as shown in Table 12, by substituting the vectors of observations from the marginal posterior distributions of $\alpha, \varepsilon$ and $\kappa$ into the quantile function in Table 2; for $0<\mathrm{F}<1$, samples from the posterior distribution of return levels can be obtained. This procedure is carried out for $\mathrm{p}=0.1,0.5$ and 0.01 , to obtain the posterior distributions of the 10-, 50- and 100year return levels.

In Table 12, an increase in extreme temperatures for the next 10, 50 and 100 years are presented, especially in S4 and S11. Table 12 exhibits an increase in extreme temperatures for the next 10, 50 and 100 years, especially in S4 and S11. This result is highly useful as a guideline for governments in implementing temperature-related policies, such as in agriculture, electricity and so on.

Table 12. Comparison of the performance of Bayesian MCMC under different return periods for GEV and GLO distributions

\begin{tabular}{cccc}
\hline Station & $\begin{array}{c}\text { Return } \\
\text { Periods 10- } \\
\text { year }\end{array}$ & $\begin{array}{c}\text { Return } \\
\text { Periods 50- } \\
\text { year }\end{array}$ & $\begin{array}{c}\text { Return Periods } \\
\text { 100-year }\end{array}$ \\
\hline S1 & 87.801 & 88.245 & 88.388 \\
S2 & 87.440 & 87.983 & 88.159 \\
S3 & 87.835 & 88.919 & 89.251 \\
S4 & 89.082 & 95.745 & 100.669 \\
S5 & 79.931 & 80.375 & 80.453 \\
S6 & 86.722 & 87.366 & 87.536 \\
S7 & 88.406 & 89.979 & 90.540 \\
S8 & 88.291 & 89.352 & 89.694 \\
S9 & 89.573 & 92.032 & 93.195 \\
S10 & 87.494 & 90.274 & 91.634 \\
S11 & 88.630 & 91.145 & 92.182 \\
S12 & 88.169 & 89.254 & 89.629 \\
\hline
\end{tabular}

\section{CONCLUSIONS}

In this study, the occurrence probability of annual maximum temperature events was analysed in the 12 stations of Sumatra Island. GEV and GLO distributions were selected to fit the data. The types of data in this study were analysed using the Bayesian MCMC techniques, particularly to estimate the parameters of the two probability distributions. In this study, Bayesian MCMC techniques worked well and efficiently with the non-informative prior distribution by validating the acceptance rate. From the results of the parameter and quantile estimations, Bayesian MCMC techniques had an advantage when the median or mean value was required. However, in the aspect of the uncertainty analysis, Bayesian MCMC can remarkably reduce the range of uncertainty. The reduction of uncertainty in the results of the frequency analysis may not always give a suitable description for all the cases. In addition, Bayesian analysis cannot always reduce uncertainty. Specifically, if we have considerable information, such as a large sample size for defining unknown parameters, then the influence of the uncertainty is relatively weak to determine a specific decision. However, if we have little information, then the analysis of the uncertainty has a strong influence on the final selection of the parameters. Therefore, reduced uncertainty in the frequency analysis with extreme events, such as the extreme temperature events in this study, can provide a meaningful description.

\section{REFERENCES}

[1] Trenberth, K.E., Shea, D.J. (2005). Relationships between precipitation and surface temperature. Geophysical Research Letters, 32(14): L14703. https://doi.org/10.1029/2005GL022760

[2] Kharin, V.V., Zwiers, F.W., Zhang, X., Wehner, M. (2013). Changes in temperature and precipitation extremes in the CMIP5 ensemble. Climatic change, 119(2): 345-357. https://doi.org/10.1007/s10584-0130705-8

[3] Munoz, A., Sánchez-Ubeda, E.F., Cruz, A., Marín, J. (2010). Short-term forecasting in power systems: A guided tour. In Handbook of Power Systems II, 129-160. https://doi.org/10.1007/978-3-642-12686-4_5

[4] Steffen, W., Hughes, L., Perkins, S. (2014). Heat waves: Hotter, longer, more often. Climate Council of Australia Limited. Second Major Technical Report of the Climate Council.

[5] Lyon, B. (2009). Southern Africa summer drought and heat waves: Observations and coupled model behavior. Journal of Climate, 22(22): 6033-6046. https://doi.org/10.1175/2009JCLI3101.1

[6] Meehl, G.A., Tebaldi, C. (2004). More intense, more frequent, and longer lasting heat waves in the $21 \mathrm{st}$ century. Science, 305(5686): 994-997. https://doi.org/10.1126/ science.1098704

[7] Noji, E.K. (2000). The public health consequences of disasters. Prehospital and Disaster Medicine, 15(4): 2131.

[8] Hahn, H., Meyer-Nieberg, S., Pickl, S. (2009). Electric load forecasting methods: Tools for decision making. European Journal of Operational Research, 199(3): 902907. https://doi.org/10.1016/j.ejor.2009.01.062

[9] Gencay, R., Selcuk, F. (2004). Extreme value theory and Value-at-Risk: Relative performance in emerging markets. International Journal of Forecasting, 20(2): 287 303. https://doi.org/10.1016/j.ijforecast.2003.09.005

[10] Sigauke, C., Verster, A., Chikobvu, D. (2012). Tail quantile estimation of heteroskedastic intraday increases in peak electricity demand. Open Journal of Statistics, 2(4): 435-442.

[11] Soares, C.G., Scotto, M.G. (2004). Application of the $r$ largest-order statistics for long-term predictions of significant wave height. Coastal Engineering, 51(5-6): 387-394. https://doi.org/10.1016/j.coastaleng.2004.04.003

[12] Gencay, R., Selcuk, F. (2004). Extreme value theory and Value-at-Risk: Relative performance in emerging markets. International Journal of forecasting, 20(2): 287303. https://doi.org/10.1016/j.ijforecast.2003.09.005

[13] Hyndman, R.J., Fan, S. (2009). Density forecasting for long-term peak electricity demand. IEEE Transactions 
on Power Systems, 25(2): 1142-1153. https://doi.org/10.1109/TPWRS.2009.2036017

[14] Kharin, V.V., Zwiers, F.W. (2005). Estimating extremes in transient climate change simulations. Journal of Climate, 18(8): 1156-1173. https://doi.org/10.1175/JCLI3320.1

[15] Kharin, V.V., Zwiers, F.W., Zhang, X., Hegerl, G.C. (2007). Changes in temperature and precipitation extremes in the IPCC ensemble of global coupled model simulations. Journal of Climate, 20(8): 1419-1444. https://doi.org/10.1175/JCLI4066.1

[16] Sterl, A., Severijns, C., Dijkstra, H., Hazeleger, W., van Oldenborgh, G.J., van den Broeke, M., van Velthoven, P. (2008). When can we expect extremely high surface temperatures? Geophysical Research Letters, 35(14): L14703. https://doi.org/10.1029/2008GL034071

[17] Jarušková, D., Rencová, M. (2008). Analysis of annual maximal and minimal temperatures for some European cities by change point methods. Environmetrics: The Official Journal of the International Environmetrics Society, 19(3): 221-233. https://doi.org/10.1002/env.865

[18] Coles, S., Tawn, J. (2005). Bayesian modelling of extreme surges on the UK east coast. Philosophical Transactions of the Royal Society A: Mathematical,
Physical and Engineering Sciences, 363(1831): 13871406. https://doi.org/10.1098/rsta.2005.1574

[19] Coles, S., Bawa, J., Trenner, L., Dorazio, P. (2001). An Introduction to Statistical Modeling of Extreme Values, 208. London: Springer. https://doi.org/10.1007/978-14471-3675-0

[20] Bernardo, J.M., Smith, A.F. (2009). Bayesian theory 405: John Wiley \& Sons.

[21] Gelman, A., Carlin, J.B., Stern, H.S., Dunson, D.B., Vehtari, A., Rubin, D.B. (2013). Bayesian Data Analysis. CRC Press.

[22] Carlin, B.P., Louis, T.A. (2000). Bayes and Empirical Bayes Methods for Data Analysis. Chapman \& Hall/CRC.

[23] Besag, J., Green, P., Higdon, D., Mengersen, K. (1995). Bayesian computation and stochastic systems. Statistical Science, 3-41.

[24] Besag, J. (2001). Markov chain Monte Carlo for statistical inference. Center for Statistics and the Social Sciences, 9: 24-25.

[25] Gamerman, D., Lopes, H.F. (2006). Markov Chain Monte Carlo: Stochastic Simulation for Bayesian Inference. CRC Press. 